\title{
Forearm extravasation injury during robot-assisted low anterior resection
}

\author{
Ji Hyun Kim, Sung Sik Park, Jong Chan Kim, Jun-Mo Park, and Sung-Hye Byun \\ Department of Anesthesiology and Pain Medicine, Kyungpook National University School of Medicine, Daegu, Korea
}

A 47-year-old female with an American Society of Anesthesiologists physical status of I was scheduled for robot-assisted low anterior resection and right hepatectomy. Her routine preoperative examination results were unremarkable. Standard monitoring devices were applied in the operating room, including a noninvasive blood pressure monitoring system, electrocardiogram, and pulse oximeter. A warm blanket was placed under the patient to maintain her body temperature. General anesthesia was induced with propofol $120 \mathrm{mg}$, continuous remifentanil infusion $0.25 \mu \mathrm{g} / \mathrm{kg} / \mathrm{min}$, and rocuronium $0.6 \mathrm{mg} / \mathrm{kg}$ via a pre-existing 20 -gauge intravenous catheter in the right ventral forearm without apparent resistance. Anesthesia was maintained with desflurane $(5-7$ vol\%) and remifentanil $(0.05-0.20 \mu \mathrm{g} / \mathrm{kg} / \mathrm{min})$. The right radial artery and right subclavian vein were catheterized. The patient was placed into the lithotomy and Trendelenburg position with both arms tucked using a fabric arm-straining device placed immediately proximal to the peripheral cannulation. All cannulations were confirmed to be functional. All fluids and medication were administered through the central cannulation with the exception of the anesthetic induction agents, $1.5 \mathrm{~L}$ of crystalloid solution (Plasmalyte ${ }^{\circledR}$; Baxter, Deerfield, IL, USA), and $250 \mathrm{ml}$ of $5 \%$ albumin, the latter two of which are isotonic and iso-oncotic, respectively, with human plasma.

Four hours later, both the arterial pressure waveform and the ipsilateral pulse oximeter reading were dampened. The monitoring devices and peripheral line flow were checked, and the peripheral line was not flowing smoothly. Because the intravenous line worked well initially and only malfunctioned later, we assumed that frequent changes in positioning might have affected the alignment or tightness of the catheter and arm-restraining device, potentially kinking or dislodging the catheter. Considering that the operation was almost completed, we immediately stopped using the peripheral intravenous line to prevent extravasation injury. A new pulse oximeter probe was applied to the patient's right ear. Because the dampening of the arterial cannulation appeared to worsen, noninvasive blood pressure was measured on the opposite arm. At the end of the procedure, the right forearm was undraped. The ventral surface of the arm was mildly swollen with bullae, redness, and skin abrasions, especially under the site of the arm-restraining device (Fig. 1). In contrast, the dorsal surface showed no abnormalities. Physical examination revealed palpable radial arterial capillary filling pressure with no sensory or motor defects. Following consulta-

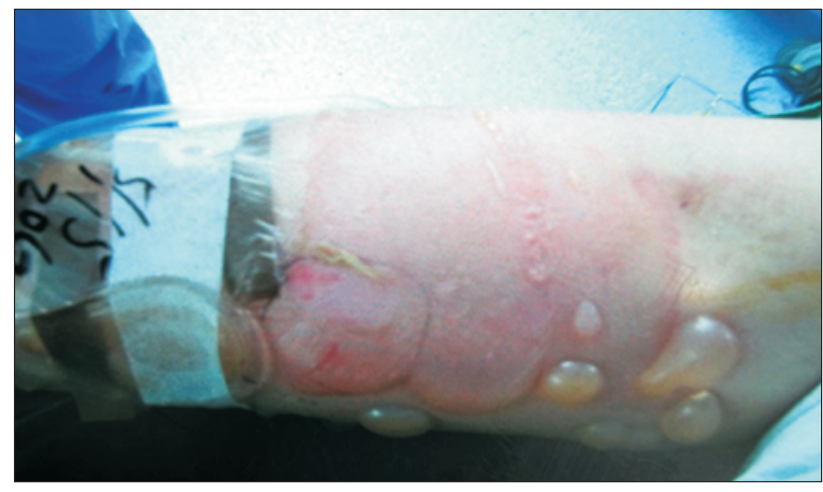

Fig. 1. Redness, skin abrasions, and bullae were observed on the ventral side of forearm once it was undraped post-operatively.

Corresponding author: Sung Sik Park, M.D., Ph.D., Department of Anesthesiology and Pain Medicine, Kyungpook National University School of Medicine, 130, Dongdeok-ro, Jung-gu, Daegu 700-721, Korea Tel: 82-53-200-5114, Fax: 82-53-426-2760, E-mail: sspark@knu.ac.kr

(c) This is an open-access article distributed under the terms of the Creative Commons Attribution Non-Commercial License (http:// creativecommons.org/licenses/by-nc/3.0/), which permits unrestricted non-commercial use, distribution, and reproduction in any medium, provided the original work is properly cited. 
tions with orthopedic and plastic surgeons, extravasation injury without compartment syndrome was diagnosed. Back-fluid was slowly aspirated from the cannula with a disposable syringe, and the catheter was carefully removed. The blisters were aspirated using 22-gauge needles under sterile conditions, and cold compression was applied.

Saline dressings were performed daily in the ward. The swelling gradually subsided, and epithelialization of the injured site was observed 2 days later. The patient was discharged with no complications.

Extravasation injury is defined as a condition in which leakage of fluid into the perivascular or subcutaneous space inadvertently occurs. It may result from various factors, including solution cytotoxicity, osmolality, and positioning [1]. In the perioperative period, such injuries are often caused by nontoxic fluids; therefore, most extravasations do not cause severe damage, in contrast to cytotoxic injury in oncologic settings. As a result, physicians underestimate the consequences of extravasation injury in the perioperative period. A few reports have described cases of extravasation injury in the perioperative period that resulted in serious complications such as compartment syndrome and tissue necrosis $[1,2]$.

In our report, although we had initially confirmed that the peripheral intravenous cannulations were functional, the function was compromised in the middle of the operation and eventually resulted in an extravasation injury. Use of the peripheral intravenous line had to be immediately stopped upon observation of evidence of extravasation or a related condition [3]. Although we could not examine the catheter when extravasation was suspected, an inference could be drawn from other conditions, and we did not hesitate to stop using the suspicious peripheral line. Fortunately, the extravasation injury did not develop into other complications such as compartment syndrome, tissue necrosis requiring fasciotomy, or debridement.

As mentioned previously, few reports contain adequate algorithms for evaluating the function of the peripheral intravenous line. According to the checklist suggested by Bebaway et al. [4], the intravenous line should be evaluated immediately after patient positioning by observing the gravity-induced free flow. This should be rechecked intermittently during the operation. Function can be confirmed upon the observation of any changes in anesthetic requirements that cannot be explained by a surgical complication or patient condition. This is done by administering a small amount of propofol $(<50 \mathrm{mg})$ or opioids $(0.3 \mu / \mathrm{kg}$ of remifentanil) and checking whether this results in a prompt increase in the depth of anesthesia.

In our case, dampening of the arterial pressure waveform and pulse oximeter reading was the first sign that we could suspect extravasation injury. Because swelling caused by extravasation can affect the arterial pulse waveform, its dampening could be important evidence of fluid extravasation as well as dampening of the pulse oximeter reading [5].

We also noted that the extravasation injury described in this report developed only around a nondependent portion. Thus, we assumed that the warm blanket under the patient may have caused vasodilatation and influenced the permeability of the vessel, accelerating inflammatory effects. Further studies are therefore warranted to determine whether and how heat affects extravasation injuries.

The detection of extravasation injuries is especially difficult when the patient is under anesthesia and unable to communicate. Procedural modifications, such as evaluating cannulae at each position change, may prevent extravasation injuries. Upon development of extravasation, immediate inspection and management are required to prevent further damage. In our report, the timely detection and management of the extravasation may have prevented more serious complications and thus improved the clinical outcome. While fluids were previously only administered by natural gravity, there has been a recent trend toward total intravenous anesthesia using a syringe pump and monofusor. Physicians must therefore be vigilant to detect and manage extravasation early.

\section{References}

1. Schummer W, Schummer C, Bayer O, Müller A, Bredle D, Karzai W. Extravasation injury in the perioperative setting. Anesth Analg 2005; 100: $722-7$.

2. Lee JA, Jeon YS, Jung HS, Kim HG, Kim YS. Acute compartment syndrome of the forearm and hand in a patient of spine surgery. Korean J Anesthesiol 2010; 59: 53-5.

3. Upton J, Mulliken JB, Murray JE. Major intravenous extravasation injuries. Am J Surg 1979; 137: 497-506.

4. Bebawy JF, Gupta DK, Koht A. Compartment syndrome caused by a properly functioning infusion pump. J Clin Anesth 2011; $23: 134-6$.

5. Kannans. Pulse oximeter aided diagnosis of fluid extravasation. Anaesthesia 2001; 56: 806-7. 